\title{
PRIMEROS RESULTADOS EN UN ENSAYO DE ESPACIAMIENTO, PODA Y RALEO CON Eucalyptus globulus EN CONSTITUCIÓN (VII REGION).
}

\author{
JUAN CARLOS PINILLA SUÁREZ
}

División Ord. Forestal e Inventarios. Instituto Forestal - Concepción.

\section{RESUMEN}

En el estudio se presentan los primeros resultados de un ensayo de 7 años de Espaciamiento. Poda y Raleo con E. globulus, realizado en el secano costero de la VII Región, utilizando distintas densidades iniciales y finales. Se observó que no existian diferencias estadisticas significativas entre las alturas de los distintos espaciamientos, lo que confirmaria que la densidad inicial no influiria sobre la altura que alcanzan los árboles dominantes. Sin embargo, se aprecia que en el crecimiento inicial el espaciamiento si tiene un efecto sobre la altura total del rodal. A una mayor densidad inicial se obtiene un menor DAP en los árboles. La mayor cantidad de área basal se obtuvo en las parcelas con una densidad intermedia de 1667 árboles por hectárea, lo que puede ser un indicador para una adecuada ocupación del sitio por parte del E. globulus, en las condiciones donde se ubica el ensayo. La disminución del número de árboles en algunas parcelas es un factor que debe incorporarse en cualquier análisis futuro del ensayo. Las relaciones funcionales obtenidas presentaron coeficientes de correlación aceptables, usando como variables independientes el DAP, la edad del rodal y la densidad del rodal.

Palabras claves: E. globulus, Densidad, Raleos, Correlaciones

\section{ABSTRACT}

The initial results of a 7 years old trial with different initial density and thinning treatments in Eucalyptus globulus are showed. This study was carried out at the Pantanillo Farm, in the VII Region (Chile), using different initial and final densities. There was not significant growth differences in the dominant height between the different densities after the second thinning. This results confirm that the initial density does not have effect in the dominant height. However, in the initial growth, after the thinning, the density had an effect on the total height of the stand. With a high initial density (2.000 trees/ha without thinning) was found the lowest d.b.h. (diameter breast height). The higher basal area was found in the treatment with an initial density of 1.667 trees/ha and a final density of 300 trees per hectar. This number of trees could indicate an adequate occupation of the site by $E$ globulus in the conditions where the trial is placed. The relations obtained had high correlations, using the d.b.h., the age and the density as independent variables.

Keywords: Eucalytus globulus, Density. Thinning, Correlations. 


\section{INTRODUCCIÓN}

Dentro del proyecto Manejo Silvícola de Especies del Género Eucalyptus, la División Ordenación Forestal e Inventarios de INFOR ha continuado la investigación destinada a incorporar distintos tipos de manejo en el cultivo del eucalipto.

El ensayo ubicado en el predio Pantanillo, perteneciente a la Universidad de Chile, es parte importante de este proyecto ya que en él se ensayan distintos tipos de espaciamientos, podas y raleos aplicados en $\mathbf{E}$. globulus. Además, el predio se ubica en un área de alto interés forestal, tanto a nivel de empresas como de propietarios particulares.

Los esfuerzos dedicados a intentar definir las labores de manejo que optimicen los objetivos de producción forestal requieren del análisis y utilización de la valiosa e interesante información que desde estos tipos de ensayo se puede extraer, incluso hasta después de la explotación del bosque. al analizar las características físicas y tecnológicas de los productos a obtener.

Los resultados son evaluados a través de las respuestas obtenidas en las variables más representativas del rodal, intentando definir y obtener las relaciones funcionales que entre ellas existen. Se intenta además obtener datos que permitan la construcción de funciones de ahusamiento y volumen aplicables a esta especie en las condiciones de sitio del ensayo.

En este trabajo se presentan los primeros resultados, para lo cual se evaluó el desarrollo actual del rodal bajo los distintos espaciamientos iniciales y el efecto de dos raleos recientes sobre él.

\section{OBJETIVOS}

\section{Objetivo General del Ensayo}

Este ensayo se enmarca dentro del proyecto Manejo Silvícola de Especies del Género Eucalyptus, cuyos objetivos principales son:

- Determinar las respuestas al manejo y los esquemas silviculturales adecuados para las especies de Eucalyptus de rápido crecimiento, en función de los objetivos de producción y las caracteristicas de cada zona edafoclimática del pais.

- Obtener datos de crecimiento y respuesta al manejo de Eucalyptus, su análisis, formulación de funciones de crecimiento y rendimiento, y su posterior aplicación práctica. 


\section{Objetivo Específico del Ensayo en el Predio Pantanillo}

Probar distintos esquemas de espaciamiento, poda y raleo en una plantación de E. globulus ssp globulus de acuerdo a un esquema predeterminado basado en el número de árboles, decidido con anterioridad, que llegará a la edad de cosecha final.

\section{MATERIAL Y MÉTODO}

\section{Área de Estudio}

El ensayo se localiza en el predio Pantanillo perteneciente a la Universidad de Chile, ubicado en la provincia del Maule. VII Región (Figura $\mathrm{N}^{\circ} 1$.).

\section{Diseño Experimental}

Este ensayo se estableció durante la temporada de plantación del año 1988 , utilizando un diseño experimental completamente aleatorizado con tres repeticiones. Se utilizaron plantas de $\mathbf{E}$. globulus a raiz desnuda. con un año de vivero, producidas en el predio.

El diseño experimental consiste de 36 parcelas, cada una de $1.936 \mathrm{~m}^{2}$ (44 $\times 44 \mathrm{~m}$ ). con un número variable de árboles en cada una, de acuerdo al espaciamiento inicial utilizado.

$\mathrm{Al}$ inicio del ensayo se establecieron los distintos regímenes de raleo y podas a ensayar, cuya aplicación se efectuaria al momento de alcanzar los árboles una altura dominante predeterminada. Este diseño se definió de acuerdo con un objetivo de producción, en el cual según el producto a obtener es el régimen a aplicar. siendo el número de árboles a ralear y la altura de poda los factores que variaban en cada tratamiento. Al combinar los distintos espaciamientos iniciales con los esquemas de poda y raleo se definieron 11 tratamientos. los que se presentan en el Cuadro $\mathrm{N}^{\circ} 1$.

La distribución del ensayo en el terreno y esquema se presentan en la Figura №2. 


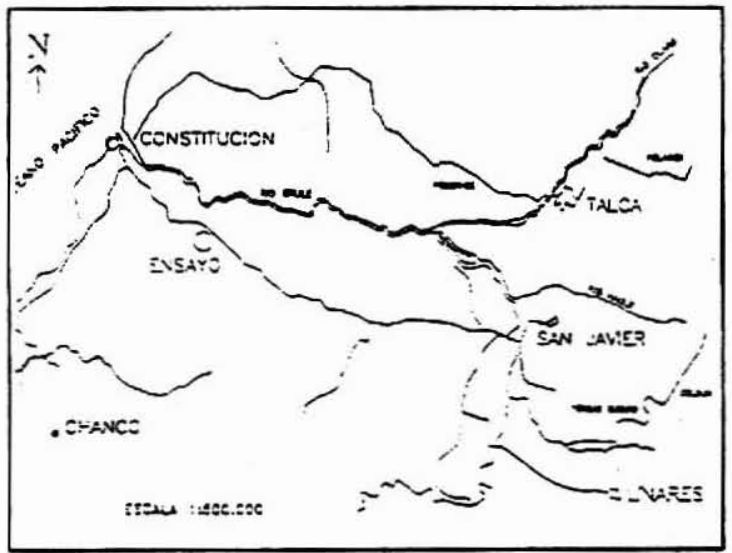

Figura No 1 . UBICACIÓN GEOGRÁFICA ENSAYO PANTANILLO

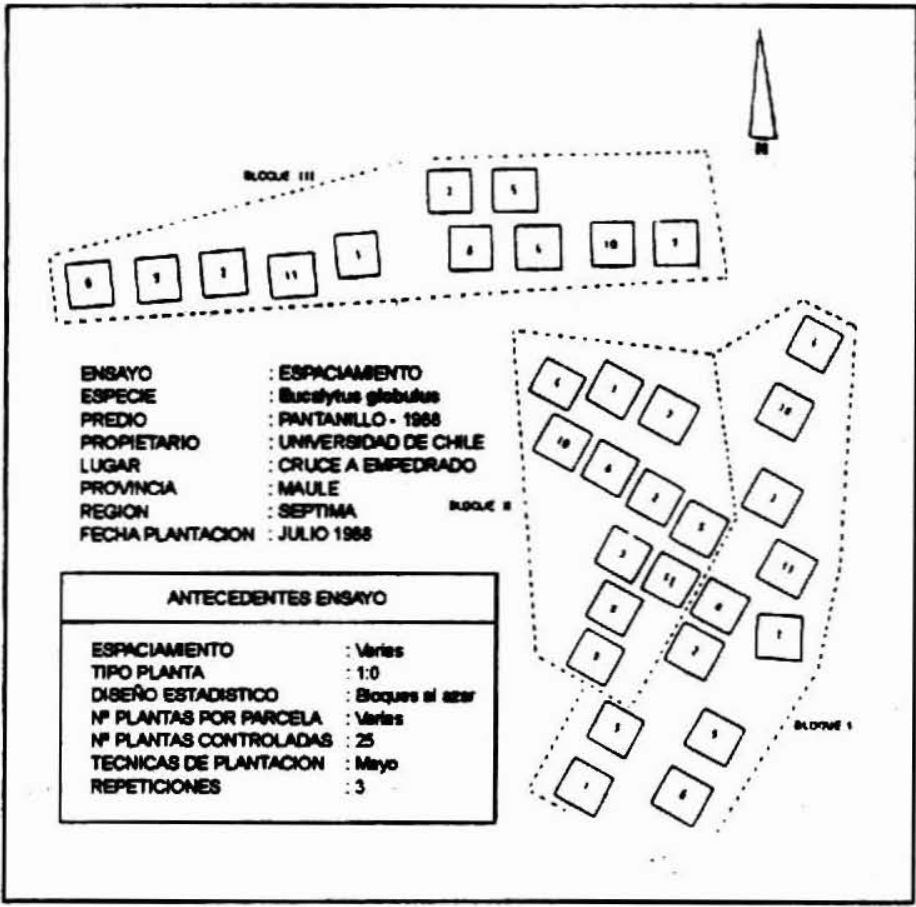

Figura $\mathbf{N}^{\circ}$ 2. DISTRIBUCIÓN DE TRATAMIENTOS 


\section{Raleos Realizados}

En el ensayo se han realizado dos raleos hasta el momento, en 1992 y 1994, de acuerdo con las alturas alcanzadas por las parcelas y el esquema del experimento. En el futuro, se deberán realizar las intervenciones finales que el diseño experimental contempla.

Cuadro $\mathrm{N}^{\mathrm{1}} 1$.

ENSAYO DE REGIMENES DE MANEJO PARA Eucalyprus globulus FUNDO PANTANILLO.

\begin{tabular}{|c|c|c|c|c|c|c|c|}
\hline \multirow{4}{*}{$\begin{array}{l}\text { Objetivos } \\
\text { Producción }\end{array}$} & \multirow{4}{*}{$\begin{array}{c}\text { Parcela } \\
\left(\mathrm{N}^{\circ}\right)\end{array}$} & \multirow{4}{*}{$\begin{array}{l}\text { Densidad } \\
\text { Inicial } \\
\text { (Arb/ha) }\end{array}$} & \multicolumn{5}{|c|}{ Altura Media (m) } \\
\hline & & & 10.5 & 10,5 & 13,5 & 13,5 & 40,0 \\
\hline & & & \multicolumn{5}{|c|}{ (Árb/ha) } \\
\hline & & & $\bar{D} / \mathrm{R}$ & PD1 & $\mathrm{D} / \mathrm{R}$ & PD2 & Cosecha \\
\hline Pulpa-Astillas & 1 & $2.500\left(^{*}\right)$ & $\ldots$ & - & $\ldots$ & - & 2500 \\
\hline Pulpa-Leña & 2 & 2500 & 1.500 & - & 400 & - & 400 \\
\hline Pulpa-Mad. aserrada & 3 & 2500 & 1.000 & 600 & 300 & 300 & 300 \\
\hline Pulpa-Mad. aserrada & 4 & 1667 & 1.000 & 600 & 300 & 300 & 300 \\
\hline Trozas exportación & 5 & $1.250\left(^{*}\right)$ & - & $\cdots$ & - & - & 1.250 \\
\hline Madera clear & 6 & 1250 & 800 & 500 & 300 & 300 & 300 \\
\hline Madera clear & 7 & 1250 & 400 & 400 & 200 & 200 & 200 \\
\hline Chapas & 8 & 833 & 500 & 500 & 300 & 300 & 300 \\
\hline Pulpa-Astillas & 9 & $625\left(^{*}\right)$ & - & 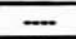 & - & - & 625 \\
\hline Madera clear & 10 & 625 & 300 & 300 & 200 & 200 & 200 \\
\hline Chapas & 11 & 625 & 300 & 300 & 300 & 100 & 100 \\
\hline
\end{tabular}

D/R: Número de árboles después del raleo.

PDI: Nủmero de árboles con altura de poda a $\operatorname{los} 3$ a $4 \mathrm{~m}$

PD2: Número de árboles con altura de poda hasta $7,5 \mathrm{~m}$.

(*) : Densidad inicial menos la mortalidad.

\section{Metodología de Análisis}

Con las mediciones efectuadas en los años 1988; 1989: 1990; 1992; 1993 y 1994 se calculó por tratamiento y bloque las siguientes variables de estado para el ensayo: DAP $(\mathrm{cm})$. Altura $(\mathrm{m})$. Altura de Copa $(\mathrm{m})$ y Arca Basal $\left(\mathrm{m}^{2} / \mathrm{ha}\right)$.

Obtenidas las variables de estado. se realizó para cada una de ellas un análisis de varianza por tratamiento. Al mismo tiempo se efectuó una prueba de Tukey, para cada uno de los análisis de varianza que mostraron significación.

En las tres primeras evaluaciones se midió el diámetro de cuello (o DAP si correspondia) y altura a 25 árboles por parcela. En 1992; 1993 y 1994 se les midió el DAP a todos los árboles de cada parcela y la altura a una muestra de 12 árboles. rectos, sanos y sin defectos. tratando que se distribuyeran de a 2 por clase diamétrica. En esta muestra se incluyó a 4 árboles de las clases diamétricas más altas. 


\section{RESULTADOS}

Las variables de estado promedio por tratamiento registradas en los distintos años de medición se presentan en el Cuadro №2.

Cuadro $\mathrm{N}^{0} 2$.

VARIABLES DE ESTADO PROMEDIO POR TRATAMIENTO

\begin{tabular}{|c|c|c|c|c|c|}
\hline TRATAMEIENTO & ANOS & $\begin{array}{l}\text { DAP MEDTlO } \\
\text { (cm) }\end{array}$ & $\begin{array}{l}\text { NLTURA MEDU } \\
(\mathrm{m})\end{array}$ & $\begin{array}{l}\text { ALTURACOPA } \\
\text { (m)(i) }\end{array}$ & $\begin{array}{c}\text { AREABASAL } \\
\left(\mathrm{m}^{2} / \mathrm{ma}\right)\end{array}$ \\
\hline 1 & $\begin{array}{l}1988 \\
1999 \\
1990 \\
1992 \\
1993 \\
1994 \\
\end{array}$ & $\begin{array}{c}0.31 \\
1.7 \\
4.47 \\
8.67 \\
9.13 \\
9.88 \\
\end{array}$ & $\begin{array}{r}0.21 \\
1.08 \\
5.05 \\
9,80 \\
14.15 \\
15,88 \\
\end{array}$ & $\begin{array}{l}\bar{F} \\
\overline{3.62} \\
4,44 \\
7.83\end{array}$ & $\begin{array}{r}0.0189 \\
0.687 \\
3.923 \\
14,739 \\
16.67 \\
19.167 \\
\end{array}$ \\
\hline 2 & $\begin{array}{l}1998 \\
1999 \\
1990 \\
1992 \\
1993 \\
1994 \\
\end{array}$ & $\begin{array}{r}0.29 \\
1.41 \\
4.35 \\
8.73 \\
9.80 \\
10.66 \\
\end{array}$ & $\begin{array}{r}0.19 \\
0.87 \\
4.89 \\
9.94 \\
12.56 \\
14.28 \\
\end{array}$ & $\begin{array}{l}\overline{7} \\
\overline{7} \\
3.59 \\
3.42 \\
7.53\end{array}$ & $\begin{array}{r}0.0165 \\
0.390 \\
3.719 \\
14.964 \\
7.43 \\
8.925\end{array}$ \\
\hline 3 & $\begin{array}{l}1998 \\
1989 \\
1990 \\
1992 \\
1993 \\
1994 \\
\end{array}$ & $\begin{array}{r}0.30 \\
1.53 \\
3.91 \\
8.63 \\
10,58 \\
11.32 \\
\end{array}$ & $\begin{array}{r}0.20 \\
0.89 \\
3,95 \\
10.20 \\
12.12 \\
13.95 \\
\end{array}$ & $\begin{array}{l}\bar{z} \\
3.65 \\
3.39 \\
6.64\end{array}$ & $\begin{array}{r}0.0177 \\
0.46 \\
3.002 \\
14.624 \\
8.791 \\
10.064 \\
\end{array}$ \\
\hline 4 & $\begin{array}{l}1969 \\
1980 \\
1990 \\
1992 \\
1999 \\
1994 \\
\end{array}$ & $\begin{array}{r}0.30 \\
1.36 \\
3.41 \\
10.29 \\
12.21 \\
13.63 \\
\end{array}$ & $\begin{array}{r}0.20 \\
0.83 \\
4.78 \\
10.44 \\
14.60 \\
16.39 \\
\end{array}$ & $\begin{array}{l}7 \\
3.51 \\
4.09 \\
6,21\end{array}$ & $\begin{array}{r}0.0118 \\
0.242 \\
3.83 \\
13,853 \\
19.907 \\
24,308 \\
\end{array}$ \\
\hline 5 & $\begin{array}{l}1969 \\
1900 \\
1990 \\
1902 \\
1993 \\
1994\end{array}$ & $\begin{array}{c}0.27 \\
2.02 \\
3.96 \\
11.03 \\
11,52 \\
12.39\end{array}$ & $\begin{array}{r}0.19 \\
1.07 \\
3.88 \\
10.44 \\
13.48 \\
14.66\end{array}$ & $\begin{array}{l}\overline{ } \\
\overline{3.56} \\
3.61 \\
6.29\end{array}$ & $\begin{array}{r}0,0072 \\
0.401 \\
1,388 \\
11.944 \\
13.029 \\
19.071\end{array}$ \\
\hline 6 & $\begin{array}{l}1998 \\
1989 \\
1990 \\
1992 \\
1993 \\
1994\end{array}$ & $\begin{array}{l}0.33 \\
1.74 \\
4.94 \\
11.00 \\
12.49 \\
13.83\end{array}$ & $\begin{array}{l}0.21 \\
1.04 \\
4.49 \\
10.50 \\
13,53 \\
14,39\end{array}$ & $\begin{array}{l}\overline{.} \\
\overline{7} \\
3,32 \\
4,05 \\
6,32\end{array}$ & $\begin{array}{r}0.0107 \\
0.297 \\
2.114 \\
11.879 \\
9.160 \\
11.267\end{array}$ \\
\hline 7 & $\begin{array}{l}968 \\
1989 \\
1990 \\
1992 \\
1993 \\
1994 \\
\end{array}$ & $\begin{array}{r}0.29 \\
1.68 \\
9.42 \\
11.06 \\
19.29 \\
17.24 \\
\end{array}$ & $\begin{array}{r}0.20 \\
0.99 \\
4.78 \\
10.88 \\
14.24 \\
15.91 \\
\end{array}$ & $\begin{array}{l}\overline{7} \\
\overline{3.81} \\
4.95 \\
6.43\end{array}$ & $\begin{array}{r}0.0083 \\
0.277 \\
2.884 \\
12.009 \\
13.699 \\
17,308 \\
\end{array}$ \\
\hline 8 & $\begin{array}{l}1988 \\
1989 \\
1990 \\
1992 \\
1993 \\
1994\end{array}$ & $\begin{array}{r}0.33 \\
1,67 \\
5.10 \\
10.38 \\
12.98 \\
14.43\end{array}$ & $\begin{array}{r}0.21 \\
0.96 \\
4.83 \\
9.99 \\
12.22 \\
13.92\end{array}$ & $\begin{array}{l}\overline{7} \\
\overline{3.95} \\
4.27 \\
9.48\end{array}$ & $\begin{array}{r}0.0071 \\
0.182 \\
1,702 \\
7,049 \\
11,022 \\
13,623\end{array}$ \\
\hline 9 & $\begin{array}{l}1998 \\
1989 \\
1990 \\
1992 \\
1993 \\
1994 \\
\end{array}$ & $\begin{array}{r}0.32 \\
1.06 \\
3.80 \\
9.75 \\
12.47 \\
14.05\end{array}$ & $\begin{array}{l}0.21 \\
0.96 \\
3.62 \\
8.68 \\
12.24 \\
13.39 \\
\end{array}$ & $\begin{array}{l}\overline{7} \\
\overline{3.71} \\
2.4 \\
4.86\end{array}$ & $\begin{array}{l}0.0050 \\
0.139 \\
0.743 \\
4.666 \\
7.633 \\
9.690\end{array}$ \\
\hline 10 & $\begin{array}{l}1988 \\
1989 \\
1990 \\
1992 \\
1993 \\
1994 \\
\end{array}$ & $\begin{array}{r}0.33 \\
1.93 \\
5.25 \\
12.21 \\
13.87 \\
18.19 \\
\end{array}$ & $\begin{array}{r}0.22 \\
1.00 \\
1.26 \\
9.63 \\
13.71 \\
19.43 \\
\end{array}$ & $\begin{array}{c}7 . . \\
\ldots .67 \\
3,26 \\
5,33 \\
\end{array}$ & $\begin{array}{r}0,0033 \\
0,183 \\
1,353 \\
7,318 \\
7,593 \\
12.993 \\
\end{array}$ \\
\hline$\pi$ & $\begin{array}{l}1988 \\
1999 \\
1900 \\
1902 \\
1993 \\
1994 \\
\end{array}$ & $\begin{array}{c}0.28 \\
1.64 \\
5.28 \\
12.48 \\
17.04 \\
19.00 \\
\end{array}$ & \begin{tabular}{r|}
0.19 \\
0.91 \\
4.19 \\
10.60 \\
14.32 \\
15.05 \\
\end{tabular} & $\begin{array}{l}\overline{7} \\
\overline{7} \\
3.75 \\
5.60\end{array}$ & $\begin{array}{r}0.0038 \\
0.132 \\
1,368 \\
7.768 \\
6.841 \\
8,906 \\
\end{array}$ \\
\hline
\end{tabular}

(1): En las 3 primeras mediciones no se evaluó esta variable. 
Al mismo tiempo, en las Figuras $\mathrm{N}^{\text {on }} 3 ; 4 ; 5 ; 6 ; 7$ y 8 se presentan los DAP, alturas y áreas basales, según cada año de evaluación y por tratamientos utilizados.

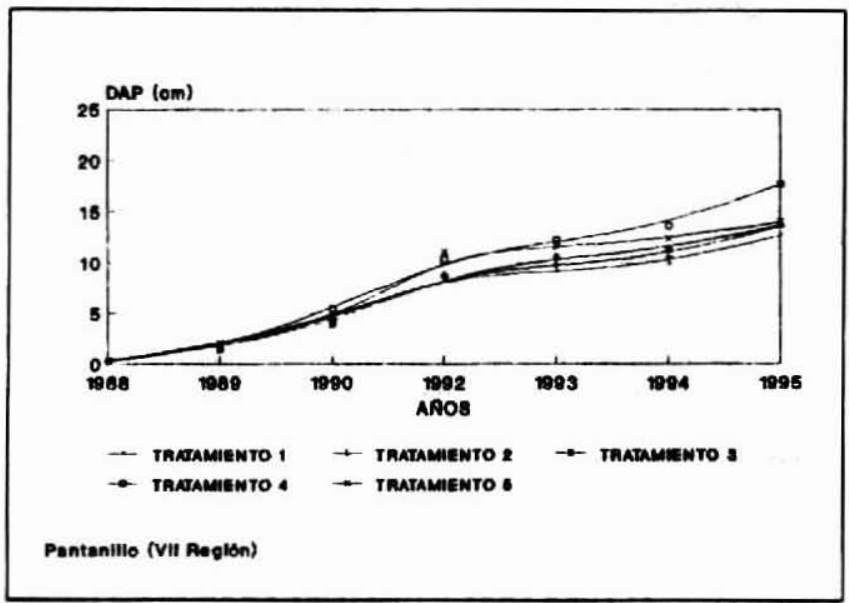

Figura No 3. DAP MEDIO TRATAMIENTOS 1 A 5 Eucalyprus globulus

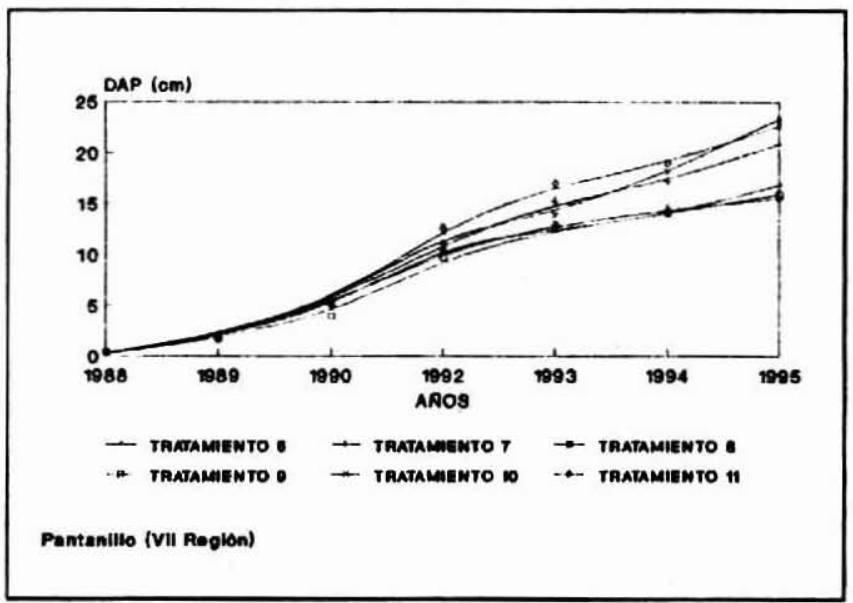

Figura $N^{\circ}$ 4. DAP MEDIO TRATAMIENTOS 6 A 11 Eucalyptus globulus 


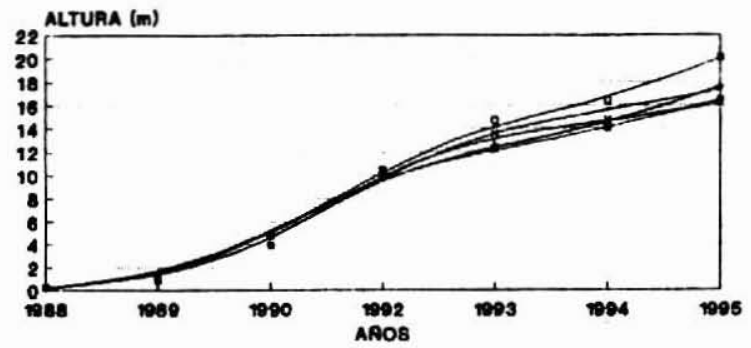

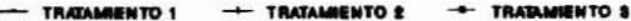

$\rightarrow$ TRMUAMENTO $4 \rightarrow$ - TRATALEENTO

Pantanflio (VII Ragion)

Figura $N^{\circ}$ 5. ALTURA TRATAMIENTOS 1 A 5 Eucalyptus globulus

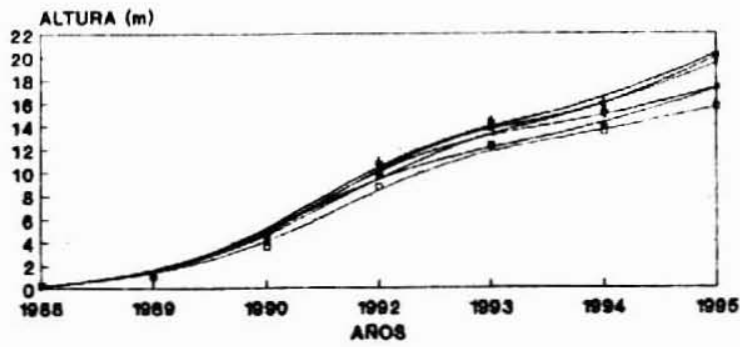

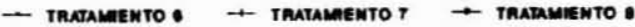

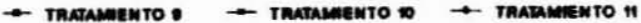

Pantanillo (VII Rogion)

Figura Nº. ALTURA TRATAMIENTOS 6 A 11 Encalyptas globulus 


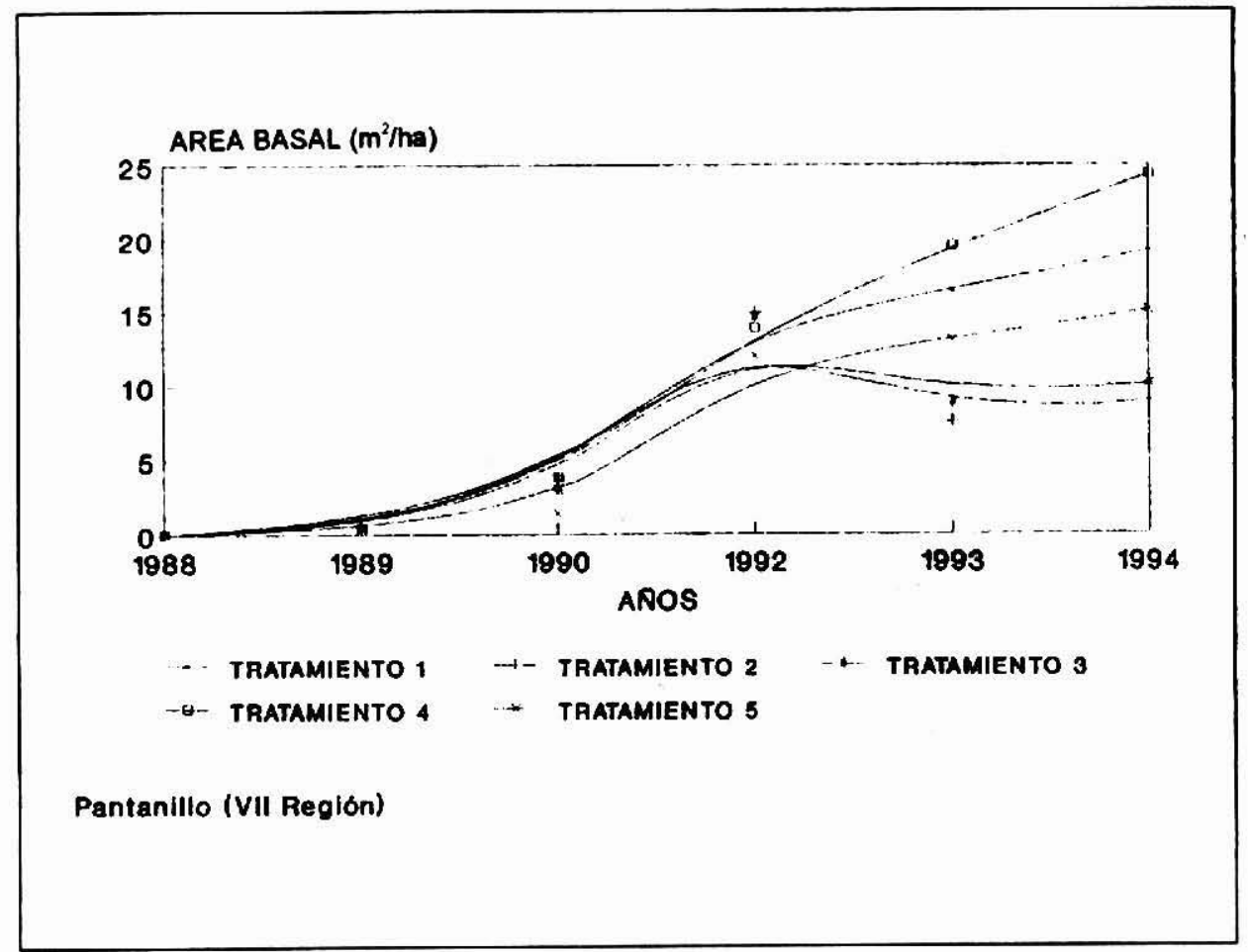

Figura $N^{\circ} 7$. AREA BASAL TRATAMIENTOS 1 A Eucalyptus globulus

Hasta la fecha se ha evaluado la información proveniente de las mediciones de 1992 y 1994. Los resultados obtenidos fueron sometidos a un análisis estadístico preliminar, analizando además el posible efecto de los recientes raleos sobre los tratamientos. Los resultados son los siguientes:

\section{Altura}

En 1992 los menores crecimientos en altura media se observan en los tratamientos de mayor espaciamiento ( $4 \times 4 \mathrm{~m}$ y $4 \times 3 \mathrm{~m}$ ), no siendo significativamente diferentes a los que se presentan en el resto de los tratamientos. Por el contrario, los mayores incrementos en altura lo presentan los tratamientos con espaciamientos de $3 \times 2$ y $4 \times 2 \mathrm{~m}$ los que, sin embargo. no son significativamente diferentes al resto de los tratamientos. 


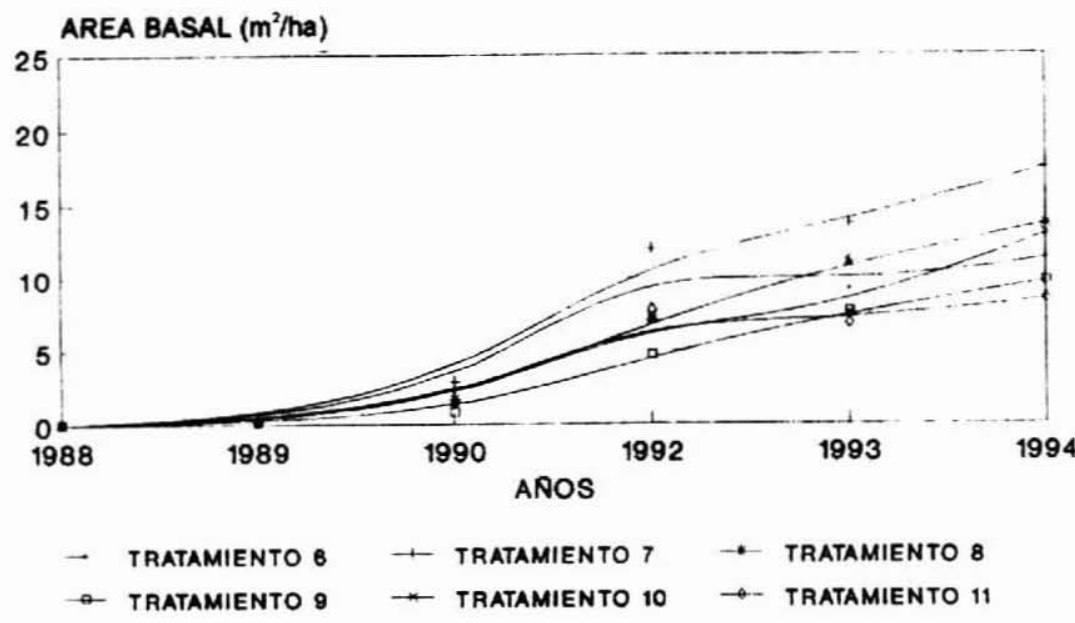

Pantanillo (VII Region)

Figura N 8. ÁREA BASAL TRATAMIENTOS 6 A 11 Eucalyptus globulus

En la medición de 1994 (Cuadro No3.), luego del raleo de 1992, se aprecia que el tratamiento 4 (1667 árboles por hectárea densidad inicial, 300 árboles por hectárea densidad final) es estadisticamente distinto en altura que los tratamientos 9 (625 árboles por hectárea densidad inicial, sin raleo), 8 (833 árboles por hectárea densidad inicial y 300 árboles por hectárea densidad final) y 3 (2.500 árboles por hectárea densidad inicial con 300 árboles por hectárea densidad final).

Este resultado, unido al efecto de una baja densidad inicial, podria ser un indicador del efecto del raleo de 1992 sobre el incremento en la altura media de los árboles, generando un mayor valor de esta variable en las parcelas intervenidas.

En el caso del eucalipto, es interesante determinar si la altura dominante es afectada significativamente por la densidad del rodal, a diferencia de lo observado en Pinus radiata en donde esta variable no es afectada por el número de árboles. 
Cuadro $\mathrm{N}^{0} 3$.

RESULTADOS EVALUACIÓN 1994

\begin{tabular}{|c|c|c|c|c|c|}
\hline \multicolumn{2}{|c|}{ DAP (cm)(1) } & Tratamiento & \multicolumn{2}{c|}{ Altura (m)(1) } \\
\hline Tratamiento & \multicolumn{2}{|c|}{ Variables } \\
\hline 1 & 9,88 & a & 9 & 13,30 & a \\
\hline 2 & 10,66 & b & 8 & 13,92 & ab \\
\hline 3 & 11,32 & b & 3 & 13,95 & ab \\
\hline 5 & 12,39 & c & 2 & 14,28 & abc \\
\hline 4 & 13,63 & d & 6 & 14,59 & abc \\
\hline 6 & 13,83 & d & 5 & 14,66 & abc \\
\hline 9 & 14,05 & d & 11 & 15,05 & abc \\
\hline 8 & 14,43 & d & 10 & 15,43 & abc \\
\hline 7 & 17,24 & e & 1 & 15,57 & abc \\
\hline 10 & 18.19 & ef & 7 & 15.91 & bc \\
\hline 11 & 19.01 & f & 4 & 16,35 & c \\
\hline
\end{tabular}

(1) : Tratamientos con igual letra no presentan diferencias significativas al nivel del $95 \%$.

En este sentido, en estas parcelas se observó que en la evaluación de 1992 no existian diferencias estadisticas significativas entre las alturas de los distintos espaciamientos. lo que confirmaria que la densidad inicial no influiría sobre la altura que alcanzan los árboles dominantes en estos rodales. Si bien no existen diferencias significativas entre las alturas alcanzadas por los árboles del estrato dominante, es claro que la altura media esta definida por la competencia con ejemplares vecinos (luz). Esto se demuestra al examinar los resultados de la evaluación de 1994. en donde sí se registraron diferencias significativas como consecuencia de la abertura del dosel.

Estos resultados indican que en los raleos que dejaron un menor número de árboles, o en las parcelas con una baja densidad inicial. se produjo en los árboles una mayor velocidad en el incremento de la altura de los árboles dominantes.

Como la evaluación del raleo de 1992 se realizó en 1994 (menos de dos años después), es posible suponer que el resultado del raleo aún no se manifiesta en toda su magnitud. o bien. que estas diferencias producidas se pueden mantener o no en el futuro. requiriendo de nuevas y sucesivas mediciones para establecer su real efecto.

\section{Diámetro a la Altura del Pecho}

Con respecto a esta variable es posible afirmar que luego de 6 años de iniciado el ensayo a una mayor densidad inicial se obtiene un menor DAP en los árboles. Es asi como el tratamiento con la mayor densidad $(2 \times 2 \mathrm{~m})$ presenta los menores incrementos en DAP en la evaluación de 1992 y es estadísticamente diferente al resto de los tratamientos. El mayor incremento diametral. en 1992, lo presentó el tratamiento con 
una menor densidad ( $4 \times 4 \mathrm{~m}$ ), el que no presenta diferencias significativas respecto a los espaciamientos de $4 \times 2,4 \times 3$ y $3 \times 2$ metros.

En la medición de 1994 el DAP presentó diferencias altamente significativas entre los distintos tratamientos. El menor valor se presentó en el tratamiento l (2500 árboles por hectárea densidad inicial sin raleo), mientras que el mayor valor estuvo en el tratamiento 11 (625 árboles por hectárea densidad inicial y 100 árboles por hectárea densidad final). En el resto de los tratamientos se presentaron valores intermedios, presentando diferencias estadísticas entre los distintos valores.

Esta mayor respuesta encontrada en los rodales de menor densidad es producto del mayor espacio con que cuentan estos individuos para la obtención de agua y nutrientes, a la mayor cantidad de luz que reciben sus copas y al mayor largo de copa registrado en estos árboles. Además se detectó una relación entre el incremento diametral y la cantidad de follaje foliar.

Esta situación podría estar significando que en las más altas densidades la competencia ya ha comenzado, estableciendo la alta concentración de DAP en las clases menores debido a la mayor cantidad de árboles presente en los tratamientos más densos.

Los resultados registrados hasta esta etapa del ensayo hacen posible concluir que a una menor densidad inicial los árboles desarrollan un mayor DAP.

Un resumen de estos resultados con su significación estadística se presentan en el Cuadro No3.

\section{Altura de Copa}

Esta variable, definida como la distancia entre la base del árbol y el inicio de su copa, presenta un comportamiento similar al de la altura total, encontrándose su mayor valor en el tratamiento con el menor espaciamiento y el menor valor en los tratamientos con la menor densidad.

El aumento o disminución de la altura de copa con respecto al DAP de los árboles permite conocer que porción del árbol (fuste o copa) influye mayormente en el crecimiento en altura, proporcionando un parámetro de apreciación sobre la aptitud comercial del árbol.

Es posible concluir, al igual que en otros estudios, que la altura de copa disminuye con el aumento del DAP, lo cual significa que la mayor proporción del incremento en altura se explica por el incremento del fuste, y que a consecuencia de ello, la contribución del fuste en la biomasa total del árbol es creciente.

El valor alcanzado por la menor densidad es producto de la menor competencia que presentan estos individuos, ya que al contar con un mayor espaciamiento reciben una mayor cantidad de luz lo que les permite una elongación de sus copas y una menor 
altura de copa. Lo contrario ocurre en las parcelas más densas, en las cuales producto del menor espaciamiento la cantidad de luz que pueden recibir los árboles es reducida en comparación a la situación anterior, por lo que sus copas, al existir una mayor competencia, no presentan la capacidad de alargarse en forma tan marcada, presentando de este modo una mayor altura de copa.

\section{Area Basal}

La mayor área basal se registró en 1994 en las parcelas con 1667 árboles por hectárea, con un valor de $24,308 \mathrm{~m}^{2} / \mathrm{ha}$, siendo interesante este dato ya que no se obtuvo en las parcelas con una mayor densidad ( 2.500 árboles por hectárea), como era de esperarse. Quizás este número de árboles por superficie sea un indicador para una adecuada ocupación del sitio por parte del E. globulus. Por otro lado, el menor valor se obtuvo en las parcelas con un mayor espaciamiento en donde la cifra llegó a los 8,506 $\mathrm{m}^{2} / \mathrm{ha}$.

Es interesante el estudio de esta variable ya que es un indicador para una situación óptima dentro del manejo silvocultural del eucalipto, en la búsqueda de la adecuada combinación de máxima área basal, mínimo número de árboles y máximos incrementos anuales en diámetro.

Es por ello que el tratamiento con la mayor densidad, a pesar de presentar una alto valor en área basal no es el óptimo desde el punto de vista del manejo forestal, ya que presenta un alto número de árboles y un DAP menor a los otros tratamientos. Por el contrario, las parcelas con el mayor espaciamiento presenta las menores cifras de área basal y número de árboles, pero sus DAP son los más altos dentro del ensayo.

Este óptimo de combinación entre número de árboles, área basal y máximo incremento depende a su vez de los productos que se quieran generar de estos bosques. Este conjunto será diferente según sea el objetivo producir madera pulpable, aserrada o cualquier otro producto.

Si el objetivo es producir madera pulpable en rotaciones cortas, no cabe duda que los tratamientos con un menor espaciamiento son los más aconsejables (alrededor de los 1667 árboles por hectárea), ya que en ellos se produce la máxima ocupación del sitio con un alto número de árboles, obteniéndose asi la mayor producción de área basal y volumen total por hectárea.

Por otra parte, si se desea obtener madera aserrada en rotaciones más largas será necesario lograr una adecuada relación entre un máximo incremento en diámetro y el número de árboles por unidad de superficie. 


\section{Funciones Predictoras}

Con los resultados obtenidos hasta el momento se intentó definir algunas relaciones funcionales que permitan predecir el comportamiento de alguna variable en función de otra. Es así como se probaron diferentes modelos para cada una de las variables de interés, como son el DAP y la altura, obteniéndose coeficientes de correlación aceptables. Estos modelos usan como variable independiente el DAP y la edad del rodal. Algunos de estos resultados se presentan en el cuadro $\mathrm{N}^{\circ} 4$.

Al intentar relacionar el espaciamiento inicial con las variables descriptoras no se obtuvieron mejores ajustes significativos. Posiblemente al agregar en este análisis el efecto futuro de los raleos sobre el desarrollo del rodal se pueda obtener alguna relación con un adecuado grado de ajuste, con el objetivo de predecir el comportamiento del DAP y de la altura en función de las variables de rodal y de la densidad.

Cuadro $\mathrm{N}^{\circ} 4$.

FUNCIONES PROBADAS EN LOS RESULTADOS DE PANTANILLO

\begin{tabular}{|c|c|c|}
\hline $\begin{array}{l}\text { Variable } \\
\text { Dependiente }\end{array}$ & Variables Independientes & $\begin{array}{l}\text { Coeficiente de } \\
\text { Correlacion }\end{array}$ \\
\hline Altura & Cle., DAP, LN DAP & 0,94 \\
\hline DAP & Cle., Edad, 1/Edad & 0,89 \\
\hline LN Altura & 1/DAP, DAP, DAP ${ }^{1 / 2}$, Densidad & 0,97 \\
\hline DAP & Edad, 1/Edad & 0,94 \\
\hline LN DAP & Cle., Edad, 1/Edad, 1/Densidad & 0,94 \\
\hline $\mathrm{DAP}^{1 / 2}$ & Edad, 1/Edad, (Edad) $)^{1 / 2}, 1 /$ Densidad & 0,98 \\
\hline DAP & Cte., Edad, 1/Edad, 1/Densidad & 0,89 \\
\hline LN AJtura & DAP, 1/Densidad, D $D^{1 / 2}, 1 /$ DAP & 0,97 \\
\hline LN Altura & Cle., 1/Edad, LN DAP, Edad. DAP', DAP, 1/DAP & 0,99 \\
\hline Altura & Cte., DAP, 1/Densidad, DAP ${ }^{2}$ & 0,93 \\
\hline Altura & Cle., DAP, DAP ${ }^{1 / 2}, 1 / \mathrm{DAP}$ & 0,92 \\
\hline Altura & Cle., DAP, 1/Densidad, 1/DAP & 0,84 \\
\hline Altura & Cte., DAP, 1/Densidad, DAP & 0,93 \\
\hline LN Altura & LN DAP, DAP. 1/DAP, DAP'. DAP ${ }^{1 / 2}$, 1/Densidad & 0,99 \\
\hline
\end{tabular}

Donde: Cte. : Constante de la función.

LN : Logaritmo natural.

DAP : Diametro a la altura del pecho.

Densidad : Densidad del rodal (arb/ha).

D : $\left(\mathrm{DAP}^{\prime}(\mathrm{DAP}+20)\right)$.

Edad : Edad en años. 


\section{Altura}

- Altura según Edad, Densidad y DAP.

Al intentar relacionar el total de las alturas de los árboles del ensayo con el diámetro a la altura del pecho y la densidad, se generaron una serie de funciones predictoras, las que luego del análisis correspondiente permiten seleccionar un modelo preliminar basado en el DAP y el efecto de la densidad.

La expresión del modelo preliminar seleccionado corresponde a:

$$
\begin{aligned}
L N H= & 0,188^{*}(D A P)-99,491^{*}(1 / D E N S I D A D)-0,758 *(D A P)^{1 / 2}+ \\
& 1,514^{*}(L N D A P)+0,197 *(1 / D A P)-0,005^{*}(D A P)^{2}
\end{aligned}
$$

$r \quad=0,986$

E.S. $=1,34$

Donde:

$H \quad$ = Altura $(\mathrm{m})$.

$D A P \quad=$ Diámetro a la altura del pecho $(\mathrm{cm})$.

$L N \quad=$ Logaritmo natural.

DENSIDAD = Densidad del rodal (arb/ha).

El modelo tiene como principal componente el resultado del DAP para explicar la altura alcanzada, hasta la última evaluación, por los árboles en el ensayo, al que se le incorpora el efecto del espaciamiento. Si bien la altura dominante que resulta no es afectada por la densidad, transcurridos siete años de este ensayo se aprecia que en el crecimiento inicial el espaciamiento sí tiene un efecto sobre la altura total del rodal.

$\mathrm{Si}$ a este análisis se le incorpora la variable edad se obtienen funciones con un mayor número de variables predictoras en su estructura, pero sin que ello signifique un aumento en el grado de ajuste. Un ejemplo de ello es la siguiente función:

$$
\begin{aligned}
& L N H=0,27 * E D A D-0,735^{*}(1 / E D A D)-0,418^{*}\left(E D A D^{1 / 2}\right)- \\
& 62,813^{*}(1 / D E N S I D A D)+1,118^{*}(L N D A P)+0,196^{*}(1 / D A P)-0,002^{*}\left(D A P^{2}\right) \\
& r \quad=0.978 \\
& E . S . \quad=1,3
\end{aligned}
$$




\section{- Altura según DAP.}

Con la información obtenida hasta el año 1994 se desarrolló una función preliminar para la estimación de la altura, basada únicamente en el DAP de los árboles, la cual se determinó según la siguiente relación:

$$
L N H=-3,109-0,418 * D A P+2,993 *(D A P)^{1 / 2}+0,002 *(D A P)^{2}
$$

$r \quad=0,98$

E.S. $=1,3$

Al examinar el comportamiento de este modelo en relación con las diferentes densidades utilizadas. se consideró necesario ajustar funciones según cada tratamiento, obteniendo con este procedimiento significativos ajustes. Las funciones obtenidas correspondieron a un modelo general con la altura o el logaritmo natural de la altura como variable dependiente y el DAP. o una de sus transformaciones, como variable independiente. La estructura general del modelo correspondió a:

$$
H(L N H)=a+b^{*} D A P+c^{*} D A P^{1 / 2}+d^{*} L N(D A P)+e^{*} D A P^{2}+f^{*}(1 / D A P)
$$

Los modelos obtenidos junto con sus respectivos coeficientes en cada tratamiento se presentan en el Cuadro $\mathrm{N}^{\circ} 5$.

\section{Cuadro $\mathrm{N}^{\circ} 5$.}

\begin{tabular}{|c|c|c|c|c|c|c|c|c|c|}
\hline \multirow{2}{*}{$T$} & \multirow{2}{*}{ VAR. } & \multirow{2}{*}{ a } & \multicolumn{5}{|c|}{ Coeficientes de cada Variable } & \multirow{2}{*}{$\mathbf{r}$} & \multirow{2}{*}{ E.S } \\
\hline & & & $\overline{\text { DAP }}$ & $\mathrm{DAP}^{1 / 2}$ & LN DAP & $\mathrm{DAP}^{2}$ & 1/DAP & & \\
\hline 1 & LN H & $-2,907$ & $-0,139$ & 2,421 & 0 & $-0,007$ & 0 & 0,99 & 1,2 \\
\hline 2 & LN H & $-3,328$ & $-0,553$ & 3,475 & 0 & 0,004 & 0 & 0,98 & 1,3 \\
\hline 3 & LN H & $-3,097$ & $-0,298$ & 2,831 & 0 & $-0,003$ & 0 & 0,98 & 1,3 \\
\hline 4 & LN H & $-2,624$ & $-0,295$ & 2,522 & 0 & 0 & $-0,078$ & 0,99 & 1,3 \\
\hline 5 & $\mathrm{LN} \mathrm{H}$ & $-2,535$ & $-0,288$ & 2,472 & 0 & 0 & $-0,083$ & 0,98 & 1,3 \\
\hline 6 & $\mathrm{LN} \mathrm{H}$ & $-3,007$ & $-0,337$ & 2,781 & 0 & 0 & 0 & 0,99 & 1,3 \\
\hline 7 & $\mathrm{LNH}$ & $-2,611$ & -0.268 & 2.405 & 0 & 0 & $-0,062$ & 0,99 & 1,2 \\
\hline 8 & $\mathrm{H}$ & $-1,318$ & 1,698 & 0 & $-0,879$ & $-0,037$ & 0 & 0,97 & 1,3 \\
\hline 9 & $\mathrm{H}$ & $-0,874$ & 1,476 & 0 & $-0,879$ & $-0,037$ & 0 & 0,96 & 1,3 \\
\hline 10 & $\mathrm{LN} \mathrm{H}$ & $-2,906$ & $-0,363$ & 2,704 & 0 & 0,002 & 0 & 0,98 & 1,3 \\
\hline 11 & LN H & -2.259 & -0.220 & 1.915 & 0.270 & 0 & 0 & 0,99 & 1,2 \\
\hline
\end{tabular}

COEFICIENTES DE LOS MODELOS SEGUN TRATAMIENTO.

Donde:

T. $=$ Tratamiento.

VAR. = Variable dependiente utilizada en el modelo.

$\mathrm{H}=$ Altura $(\mathrm{m})$.

LN H = Logaritmo natural de la altura. 


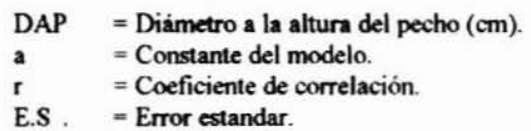

\section{- Altura según Año de Medición}

Una vez obtenidos estos modelos se procedió a ajustar funciones según cada año de medición, con el objetivo de relacionar la edad del rodal al momento de la medición, y en algunos casos la variable densidad, con el crecimiento en altura, consiguiendo también con este análisis significativos ajustes.

Las funciones obtenidas correspondieron a un modelo general del tipo:

$$
\begin{gathered}
H(L N H)=k+a^{*} D A P+b^{*}(L N D A P)+c^{*}\left(D A P^{2}\right)+d^{*} \\
(I D E N S I D A D)+e^{*}\left(D A P^{I / 2}\right)+f^{*}(I / D A P)+g^{*}(D E N S I D A D)+h^{*}\left(D^{1 / 2}\right)
\end{gathered}
$$

Donde:

$$
\begin{array}{ll}
H & =\text { Altura }(\mathrm{m}) \\
D A P & =\text { Diámetro a la altura del pecho }(\mathrm{cm}) . \\
L N & =\text { Logaritmo natural. } \\
D E N S I D A D & =\text { Densidad del rodal (arb/ha). } \\
D & =(\mathrm{DAP} /(\mathrm{DAP}+20)) . \\
k & =\text { Constante del modelo. }
\end{array}
$$

$a, b, c, d, e, f, g, h=$ Coeficientes de regresión del modelo.

\begin{tabular}{|c|c|c|c|c|c|c|c|c|c|c|c|}
\hline \multirow{2}{*}{ 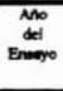 } & \multirow{2}{*}{ 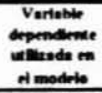 } & \multicolumn{9}{|c|}{ 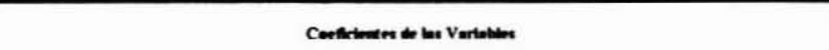 } & \multirow{2}{*}{ Coeflicient } \\
\hline & & $\mathrm{k}$ & 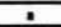 & $b$ & c & 8 & $\div$ & 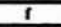 & 1 & $m$ & \\
\hline 1 & $\mathrm{H}$ & 0 & $-2,408$ & 0.898 & 2.069 & 0 & 0 & 0 & 0 & 0 & 0.99 \\
\hline 2 & H & 0.146 & 0.550 & 0 & 0,02 & .39 .003 & 0 & 0 & 0 & 0 & 0,93 \\
\hline 3 & H & -12.5 & $-1,481$ & 0 & $\overline{0}$ & 0 & 10.72 & 3.644 & 0 & 0 & 0.92 \\
\hline 5 & $\mathrm{H}$ & 10.208 & 0.292 & 0 & 0 & .1421 .87 & 0 & -17.49 & 0 & 0 & 0.84 \\
\hline 6 & LNH & 0 & -0.087 & 0 & 0 & 0 & 0 & 1.014 & 0,006 & 0,948 & 0.99 \\
\hline 7 & LNH & $\overline{0}$ & .0 .113 & 0 & 0 & -60.802 & 0 & 3.273 & 0 & 1.099 & 0.99 \\
\hline
\end{tabular}

Los coeficientes según cada año de medición se presentan en el Cuadro $\mathrm{N}^{\circ} 6$

Al observar estas funciones se aprecia que además del DAP como principal variable predictora de la altura, la densidad esta involucrada en la mayoria de los modelos obtenidos, siendo significativa para determinar el crecimiento en altura de los árboles hasta esta etapa del ensayo.

\section{Cuadro No6.}

COEFICIENTES DE LAS VARIABLES SEGÚN LA EDAD. 


\section{- Altura según Tratamiento y Edad}

En relación con la edad del ensayo no fue posible obtener ajustes significativos al relacionar la altura de los árboles únicamente con la edad del ensayo, debido posiblemente al efecto de las densidades iniciales utilizadas. Se emplearon modelos lineales y no lineales para describir esta relación entre la edad y la altura, los que presentaron ajustes significativos. pero luego del análisis de su comportamiento se concluyó que no eran explicativos del comportamiento de la altura. Una de las relaciones de forma no lineal obtenidas correspondió a:

$$
H=14,99 /\left(1+57,315^{*} \operatorname{EXP}\left(-1,026^{*} E\right)\right)
$$

$$
\begin{aligned}
r & =0.95 \\
E . S . & =0.993
\end{aligned}
$$

Donde:

$$
\begin{aligned}
H & =\text { Altura }(\mathrm{m}) . \\
E & =\operatorname{Edad}(\text { años) }
\end{aligned}
$$

En este mismo sentido. en cl análisis se intentó relacionar la altura con la edad del rodal. ajustando funciones según el tratamiento. considerando las diferentes densidades iniciales y raleos realizados. El resultado de este procedimiento fue una función general para los 11 tratamientos. cuya estructura es:

$$
H=a+b^{*}(\text { Edad })+c^{*}(1 / \mathrm{Edad})
$$

Los cocficientes resultantes en cada tratamiento se presentan en el cuadro $\mathrm{N}^{\circ} 7$.

$$
\text { Cuadro } \mathrm{N}^{\mathrm{0} 7} \text {. }
$$

\section{COEFICIENTES SEGÚN TRATAMIENTOS}

\begin{tabular}{|c|c|c|c|c|c|}
\hline \multirow{2}{*}{ Tratamientos } & \multicolumn{3}{|c|}{ Coeficientes de Regresión } & \multirow{2}{*}{ Coeficiente de Correlación } & Error Standar \\
\cline { 2 - 4 } & $\mathrm{a}$ & $\mathrm{b}$ & $\mathrm{c}$ & 0.95 & 1,5 \\
\hline 1 & -4.353 & 2.883 & 1.524 & 0.94 & 1.6 \\
\hline 2 & -4.532 & 2.765 & 1,775 & 0.95 & 1,6 \\
\hline 3 & -4.718 & 2,729 & 2.024 & 0,97 & 1,4 \\
\hline 4 & $-6,834$ & 3,348 & 3,552 & 0.96 & 1,6 \\
\hline 5 & -6.160 & 3,069 & 3,182 & 0,95 & 1,6 \\
\hline 6 & $-3,771$ & 2,724 & 1.087 & 0,96 & 1,6 \\
\hline 7 & -4.824 & 3.037 & 1.826 & 0.95 & 1,6 \\
\hline 8 & -3.926 & 2.609 & 1.354 & 0,94 & 1,6 \\
\hline 9 & -5.181 & 2.731 & 2.562 & 0.96 & 1.6 \\
\hline 10 & -5.962 & 3.074 & 3.001 & 0.97 & 1.5 \\
\hline 11 & -5.543 & 3.064 & 2.526 & & \\
\hline
\end{tabular}




\section{Diámetro a la Altura del Pecho}

Al intentar relacionar esta variable con la edad y densidades del ensayo se obtuvo una serie de funciones, las cuales si bien no presentaron altos ajustes, permiten establecer cierto grado de correlación. El modelo preliminar seleccionado en esta etapa del ensayo correspondió a la siguiente expresión:

$$
\begin{gathered}
D A P^{1 / 2}=0,186^{*}(E D A D)- \\
0,777^{*}(1 / E D A D)+0,962^{*}\left(E D A D^{1 / 2}\right)+150,035^{*}(1 / D E N S I D A D)
\end{gathered}
$$

$r \quad=0,979$

E.S. $=0,46$

Donde:

$D A P=$ Diámetro a la altura del pecho $(\mathrm{cm})$.

$E D A D=$ Edad (años).

DENSIDAD $=$ Densidad del rodal (arb/ha).

\section{- Area Basal}

Esta variable se relacionó con la edad y densidad del ensayo seleccionándose en forma preliminar para esta etapa del proyecto el siguiente modelo:

$$
\begin{gathered}
A B=-0,0103+0,0041^{*} E D A D+0,008 I^{*}(I / E D A D)- \\
0,00000138^{*}(D E N S I D A D)
\end{gathered}
$$

$\begin{aligned} r & =0,80 \\ E . S . & =0,0047\end{aligned}$

Donde:

$A B=$ Area basal $\left(\mathrm{m}^{2} / \mathrm{ha}\right)$.

$E D A D=$ Edad (años).

DENSIDAD $=$ Densidad del $\operatorname{rodal}(\mathrm{arb} / \mathrm{ha})$.

- Altura de Copa.

El comportamiento de esta variable en relación a su mayor valor al aumentar la densidad y su disminución al aumentar el DAP, podría indicar su dependencia de estas 
dos variables. Sin embargo, al realizar el análisis respectivo se encontró que sólo la altura y el logaritmo natural de ella, eran estadisticamente significativas para predecir la altura de copa.

El modelo general seleccionado en forma preliminar en el ensayo correspondió a:

$$
H C=.0,140412^{*}(H)+0,830584^{*}(L N H)
$$

$\begin{array}{ll}r & =0,94 \\ \text { E.S. } & =1,4\end{array}$

Donde:

$H C=$ Altura de copa $(\mathrm{m})$.

$H=$ Altura Total $(\mathrm{m})$.

$L N=$ Logaritmo natural.

\section{CONCLUSIONES}

Luego de transcurridos 7 años de establecido el ensayo se pueden obtener algunas conclusiones a nivel preliminar. sin considerar el real efecto del raleo sobre el desarrollo de los árboles. Estas corresponden a:

Los menores crecimientos en altura media se observan en los tratamientos de mayor espaciamiento ( $4 \times 4 \mathrm{~m}$ y $4 \times 3 \mathrm{~m}$ ), no siendo significativamente diferentes a los que se presentan en el resto de los tratamientos.

Los mayores incrementos en la altura media de los árboles, en la evaluación de 1992, lo presentan los tratamientos con espaciamientos de $3 \times 2$ y $4 \times 2 \mathrm{~m}$ los que, sin embargo, no son significativamente diferentes al resto de los tratamientos.

En la evaluación de 1994, luego del raleo de 1992, se aprecia que el tratamiento 4 (1667 árboles por hectárea densidad inicial, 300 árboles por hectárea densidad final) es superior y estadisticamente distinto en altura que el resto de los tratamientos, incluidos los de un mavor espaciamiento final..

Este resultado. unido al efecto de una baja densidad inicial, podria ser un indicador del efecto del raleo de 1992 sobre el incremento en la altura media de los árboles, generando un mayor valor de esta variable en las parcelas intervenidas hasta esta etapa del ensavo.

En estas parcelas se observó que no existian diferencias estadísticas significativas entre las alturas de los distintos espaciamientos, lo que confirmaría que la densidad inicial no influiria sobre la altura que alcanzan los árboles dominantes en estos rodales. Como la evaluación del raleo de 1992 se realizó en 1994 (dos años después), es posible suponer 
que el resultado del raleo aún no se manifiesta en toda su magnitud, requiriendo de nuevas y sucesivas mediciones para establecer su real efecto.

Luego de 7 años de iniciado el ensayo a una mayor densidad inicial se obtiene un menor DAP en los árboles.

El tratamiento con la mayor densidad, (tratamiento $1,2 \times 2 \mathrm{~m}$ ), presenta los menores incrementos en DAP y es estadisticamente diferente al resto de los tratamientos.

El mayor valor en diámetro lo presentó el tratamiento con una menor densidad ( $4 \times 4$ $\mathrm{m}$ ), el que no presenta diferencias significativas respecto a los espaciamientos de $4 \times 2$, $4 \times 3$ y $3 \times 2$ metros, siendo un resultado importante en la definición de densidades óptimas para la utilización del sitio.

En el resto de los tratamientos el DAP presentó valores intermedios, mostrando diferencias estadísticas significativas entre los distintos valores. Esta mayor respuesta encontrada en los rodales de menor densidad puede ser producto del mayor espacio con que cuentan estos individuos para la obtención de agua y nutrientes; a la mayor cantidad de luz que reciben sus copas y al mayor largo de copa registrado en estos árboles.

Es posible concluir que en los tratamientos con las mayores densidades la competencia ya ha comenzado. debido a la mayor concentración de DAP en las clases menores, al menor largo de copa registrado y al cierre de copas producido.

Los resultados registrados hasta esta etapa del ensayo hacen posible concluir que a una menor densidad inicial los árboles desarrollan un mayor DAP.

La altura de copa presenta un comportamiento similar al de la altura total, encontrándose su mayor valor en el tratamiento con el menor espaciamiento y el menor valor en los tratamientos con un menor número de árboles.

Es posible concluir que la altura de copa disminuye con el aumento del DAP, lo cual significa que la mayor proporción del incremento en altura se explica por el incremento del fuste. El valor alcanzado por la menor densidad es producto de la menor competencia que presentan estos individuos, ya que al contar con un mayor espaciamiento reciben una más alta cantidad de luz lo que les permite una elongación de sus copas.

La mayor área basal se encuentra en las parcelas con 1667 árboles por hectárea. Posiblemente, este número de árboles por superficie sea un indicador para una adecuada ocupación del sitio por parte de $\mathbf{E}$. globulus. Por otro lado, el menor valor se obtuvo en las parcelas con un mayor espaciamiento. El tratamiento con la mayor densidad a pesar de presentar una alto valor en área basal no es el óptimo desde el punto de vista del manejo forestal, ya que se presenta en una situación de alto número de árboles con DAP menores en relación a otras densidades. 
Hasta esta etapa del ensayo se puede concluir que los tratamientos con un menor espaciamiento (alrededor de los 1667 árboles por hectárea) son los más aconsejables para producir madera pulpable en rotaciones cortas, ya que en ellos se produce la máxima ocupación del sitio con un adecuado número de árboles.

Las relaciones funcionales obtenidas presentaron coeficientes de correlación aceptables, usando como variables independientes el DAP, la edad del rodal y la densidad del rodal.

Es posible que al agregar en este análisis el efecto de los raleos sobre el desarrollo del rodal se pueda obtener alguna relación con un adecuado grado de ajuste, entre el comportamiento del DAP y de la altura en función de las variables de rodal y de la densidad. .

Si bien la altura dominante que resulta no es afectada por la densidad, transcurridos siete años de este ensayo se aprecia que en el crecimiento inicial el espaciamiento si tiene un efecto sobre la altura total del rodal.

Estos resultados corresponden a una fase intermedia del ensayo, por lo que si bien pueden ser utilizados como una descripción de la situación actual del experimento, deberán ser complementados con el análisis del efecto de los raleos sobre las variables descriptoras de rodal. La disminución del número de árboles en algunas parcelas es un factor que debe incorporarse en cualquier análisis futuro del ensayo.

\section{REFERENCLAS BIBLIOGRÁFICAS}

FAO., 1980. Estimación del Volumen Forestal y Predicción del Rendimiento. Vol. 22/1 y $22 / 2$. Roma. $80 \mathrm{p}$.

Hughell, D., 1990. Modelos para La Predicción del Crecimiento y Rendimiento de Eucalyptus camaldulensis en América Central. CATTE, 1990. Boletin Técnico N22.

INFOR., 1991. Eucalyptus, Principios de Silvicultura y Manejo. CORFO-INFOR, Santiago. 197 p.

INFOR., 1994. Informe de Avance Ensayo Espaciamiento, Poda y Raleo con Eucalyptus globulus. División Ordenación Forestal e Inventarios. Documento Interno. 13 p.

Kerruish, C., and Rawlins, W., 1991. The Young Eucalypt Report. CSIRO, Australia, 272 p.

Ostle, B., 1973. Estadistica Aplicada. México. Ed. Limusa-Willey. 629 p. 